\title{
Uma leitura sobre a perspectiva etnoepidemiológica
}

\author{
A paper about ethnoepidemiologic \\ perspective
}

Rita de Cássia Pereira Fernandes 1

1 Instituto de Saúde Coletiva, UFBA. Rua Padre Feijó, s/n, Canela, 40110-170 Salvador BA.

ritapfernandes@uol.com.br
Abstract The contribution of epidemiology to the study of diseases and their distributions in populations is undoubtless. However, many experts have pointed out the necessity to take into account the difficulties and limitations of this discipline. They call the attention to the necessity of integrating different theoretical and methodological perspectives to the study of complex objects like the health-disease relationship. The limits of the risk factors approach, when isolated applied to the study of such relationship, must be surpassed by means of models able to address the relationship among human subjects and their cultural and socio-historical environment. The meeting of the epidemiological method, which is fully adequate for generalization, with another method, able to deepening, can be reached by means of the ethnoepidemiological strategy. This strategy assumes that health-disease phenomena are social processes which must be taken as such: historical, complex, phragmented, conflictive, dependent, ambiguous and uncertain. This essay aims to analyze some theoretical and methodological aspects which may allow a reflection about ethnoepidemiology potentialities, as an approach which integrates epidemiology to other disciplines.

Key words Ethnoepidemiology, Epidemiologic theory, Epidemiology limits, Discipline integration
Resumo É indubitável a contribuição da epidemiologia para o estudo da enfermidade e sua distribuição em populações. No entanto, vários estudiosos têm apontado a necessidade de revisar as dificuldades e limites dessa disciplina. Alguns autores chamam a atenção para a necessidade de integrar diferentes perspectivas teórico-metodológicas para o estudo de objetos complexos como a relação saúde-doença. Os limites da abordagem dos fatores de risco aplicada isoladamente ao estudo dessa relação devem ser superados por meio de modelos capazes de abordar a relação entre sujeitos humanos e seu meio, ambiente, cultural e sóciohistórico. O encontro entre o método epidemiológico, privilegiado para generalização, e outro capaz de aprofundamento pode ser alcançado pela estratégia etnoepidemiológica que tem como pressuposto que os fenômenos da saúde-doença são processos sociais, devendo ser concebidos como tais, "históricos, complexos, fragmentados, conflitivos, dependentes, ambíguos e incertos". Este ensaio pretende analisar alguns aspectos teórico-metodológicos que têm permitido a reflexão sobre as potencialidades da etnoepidemiologia, como abordagem que integra a epidemiologia a outras disciplinas.

Palavras-chave Etnoepidemiologia, Teoria epidemiológica, Limites da epidemiologia, Integração disciplinar 


\section{Introdução}

A epidemiologia é reconhecida como a ciência que estuda a distribuição e freqüência de doença nas populações. Seu método tem permitido investigar como a doença se comporta em uma determinada população e revelar, algumas vezes, porque a distribuição se faz de forma desigual. Ou seja, através desse método têm sido estudados os riscos epidemiológicos desiguais entre parcelas da população. Aliás, esse é o verdadeiro objeto da epidemiologia, tendo em vista que se as populações apresentassem perfis epidemiológicos homogêneos não haveria motivo para o estudo epidemiológico, que se caracteriza por tentar esclarecer o porquê das diferenças na distribuição do agravo.

Parece unânime o reconhecimento da importante contribuição da epidemiologia no estudo de agravos em população. A produção científica dos epidemiologistas atesta, indubitavelmente, a história dessa ciência da saúde (ou da doença). Nas mais diversas áreas do conhecimento em saúde, a aplicação do método epidemiológico tem conseguido fornecer respostas valiosas, as quais têm subsidiado intervenções tanto no plano das políticas públicas do setor saúde, quanto no plano individual, nas intervenções preventivas e terapêuticas.

O lugar alcançado pela epidemiologia no estudo dos fenômenos de saúde-doença em populações e, particularmente, o alcançado pelo seu método, especialmente alicerçado na matemática - embora tenha também como pilares, além da estatística, as ciências sociais e a clínica, para usar um conceito de Pereira (1995) -, conferiu à disciplina o privilégio de autoridade no estatuto científico hegemônico. Isso porque, ao se basear, prioritariamente, na objetividade conferida pelos métodos estatísticos, a epidemiologia atende a esse pressuposto, da objetividade, ainda tão almejado na ciência. Tanto é assim que inúmeras vezes observam-se pesquisadores, que utilizam os métodos qualitativos, fazerem referência à validação dos seus resultados através de medidas quantitativas, muitas vezes reunidas sem qualquer rigor metodológico, mas que supostamente atenderiam à objetividade da ciência. Dessa forma, na busca do reconhecimento do estatuto científico, os pesquisadores importam critérios de validade do método epidemiológico ou mostram resultados quantitativos, que em nada contribuem para o corpo de conhecimento produzido com base em uma metodologia qualitativa. Um exemplo é relatado em um capítulo do livro de Arthur Kleinman intitulado "The new wave of ethnographies in medical anthropology" (1995) no qual o autor apresenta sua crítica a diversas etnografias. Ao criticar aspectos metodológicas de um texto de Libbet Crandon-Malamud sobre o pluralismo médico nas montanhas bolivianas, Kleinman pondera como sendo desnecessária a apresentação de dados quantitativos que, aliás, são obtidos de forma precária e, sobretudo, porque a eles é atribuído o papel de convencer o leitor de que as conclusões da etnografia são realmente válidas.

Sevalho \& Castiel (1998) enfatizam que através da linguagem estatística a epidemiologia busca investigar comparativamente a distribuição da doença e o risco de adoecer e, a partir das associações encontradas, orientar a aplicação de medidas de controle. No entanto, os autores questionam até onde essas medidas voltadas para o controle de fenômenos complexos, como os eventos de saúde, identificados sem considerar a singularidade do adoecer humano, poderão obter respostas mais satisfatórias.

Um outro pilar da epidemiologia, conforme já referido, é representado pela clínica. Uma das mais fortes críticas formuladas pelos cientistas sociais à clínica, ou melhor, à biomedicina, conforme tratam os antropólogos, diz respeito ao lugar de autoridade, absoluta e soberana, ocupado pelo médico na prática do diagnóstico e tratamento daquele a quem denomina paciente. Não sendo interesse deste ensaio discutir o estatuto da clínica, a prática médica e as relações de poder envolvidas nesta, a referência à clínica como um dos pilares da epidemiologia servirá apenas para identificar uma das características desta última, que parece advir desse pilar, que é a exclusão da percepção do sujeito acerca do seu processo de saúde-doença na construção do saber epidemiológico. É conhecido que a prática da biomedicina se assenta hegemonicamente na negação do saber do sujeito que adoece. Esse doente fornece dados sobre sintomas, o médico obtém dados objetivos ao exame físico e formula um diagnóstico, cabível na taxonomia clínica, explicado exclusivamente pela biomedicina. Para Barreto \& Alves, embora o objeto da clínica seja idealisticamente definido como a doença no indivíduo, o seu real objeto é a doença, em estruturas biológicas (1994).

A epidemiologia, então, em nome da objetividade do método e, sem dúvida, em função de lidar com população e, portanto, restringir- 
se à abordagem horizontal, não aprofundada dos indivíduos (quantitativa, em contraposição à abordagem vertical, do estudo em profundidade, qualitativo), construiu uma grande distância entre o saber acerca do indivíduo, sua unidade de análise, e o sujeito que adoece, que ocupa apenas o lugar de objeto do conhecimento epidemiológico (Dunn \& Janes, 1986). Aqui, a epidemiologia reproduz o modelo biomédico, excluindo o sujeito, sua cotidianidade e sua história.

O pilar representado pelas ciências sociais pode ser problematizado, como o fazem Barreto \& Alves (1994). Para esses autores, o coletivo na epidemiologia toma uma forma fundamentalmente estatística, baseando-se na suposição de que existe uma entidade definida, em termos de um sistema objetivo de relações sociais, à parte das manifestações pessoais dos indivíduos. $\mathrm{E}$ mais: os indivíduos são reduzidos a unidades constitutivas de uma população e devem ser tratados como unidades mensuráveis e independentes (op. cit.).

Para muitos estudiosos, embora seja uma ciência muito jovem, a epidemiologia progrediu muito no desenvolvimento de estratégias e técnicas de pesquisa, que permitiram a vasta produção de conhecimento no seu curto tempo de vida. Na verdade, isso é creditado à necessidade que a disciplina apresentava de se afirmar institucionalmente, dando respostas e demarcando as fronteiras do seu campo de conhecimento. A esse salto metodológico, no entanto, parece não ter correspondido um acúmulo teórico da epidemiologia, sendo esta uma das suas fragilidades (Almeida Filho, 2000).

Para Sevalho e Castiel (1998), há necessidade de integrar novas perspectivas ao método epidemiológico, tendo em vista os limites das ações de saúde baseadas apenas na epidemiologia. O descolamento cultural e o desconhecimento das representações de saúde e doença, que caracterizam o método epidemiológico, repercutem nos resultados das ações de controle. Para os autores, encarar o adoecer humano como objeto indisciplinado de estudo pode possibilitar transitar entre a enfermidade em populações e as moléstias e suas representações nos grupos socioculturais, a fim de promover intervenções menos insatisfatórias do que as existentes no campo biomédico-epidemiológico atual (op. cit.).

$\mathrm{Na}$ sua empreitada de compreender a epidemiologia, desconstruindo-a para melhor construí-la, Almeida Filho tem sistematizado a proposta da etnoepidemiologia, embora outros autores também tenham feito uso prévio deste termo. Artigo publicado em 1991, por Wilbert \& Haiek, acerca de um screening fitoquímico realizado em plantas utilizadas para tratamento de desordens gastrointestinais por povos do delta do Orinoco, na Venezuela, faz uso de um protocolo de pesquisa denominado etnoepidemiológico. O termo etnoepidemiologia foi identificado também em artigo publicado em 1992, por Kunstadter et al., sobre as causas e conseqüências do aumento das taxas de sobrevida em crianças na Tailânida. Deste ano, data o primeiro artigo de Almeida Filho que foi acessado e que é referido neste ensaio. Para este autor, a etnoepidemiologia é entendida por ele, desde seus primeiros escritos, como uma disciplina que não será uma mera aplicação de métodos epidemiológicos à pesquisa transcultural em saúde, nem a introjeção de etno-modelos dentro de estruturas de explicação baseadas na abordagem de risco. A etnoepidemiologia poderá dedicar-se a explorar alternativas metodológicas para a pesquisa sobre processos e práticas sociais ligadas à saúde, aptas a combinar de modo competente as abordagens qualitativas e quantitativas em uma única estratégia etnoepidemiológica (1992).

Embora o debate sobre as possibilidades de integração entre a epidemiologia e a antropologia já tenha produzido uma importante literatura, chegando alguns a afirmar que o termo "epidemiologia comportamental" tenha sido usado desde os anos 70, este texto não é uma revisão sistemática sobre as contribuições existentes acerca da integração da epidemiologia a outras disciplinas (Janes, Stall \& Gifford, 1986; Sallis, Owen \& Fotheringham, 2000). No entanto, pretende investigar aspectos teórico-metodológicos, a partir de alguns autores que têm permitido a reflexão sobre as potencialidades de uma abordagem que integre disciplinas para o estudo dos fenômenos de saúde. Dentre as abordagens possíveis, optou-se por revisar o que tem sido sistematizado em torno de uma etnoepidemiologia.

\section{A epidemiologia no centro de um debate}

Em um texto escrito em 1992, Bibeau, apresentando uma proposta de metodologia alternativa para a antropologia médica, sinaliza o que, segundo ele, demarcaria as diferenças de perspectiva entre a epidemiologia e a antropologia. 
Em primeiro lugar, diz que a epidemiologia toma para estudo a enfermidade, ao invés dos problemas em populações. Parte de uma definição profissional do que seja enfermidade, em detrimento de uma definição popular ou do sistema de signos. Utiliza categorias médicas, supondo geralmente uma causalidade linear com limitado número de fatores determinantes. Por sua vez, a antropologia, segundo Bibeau, lida com categorias menos definidas, supondo uma causalidade mais global. Em vez de se ater às taxas de prevalência de enfermidades e sua precisão quantitativa, a antropologia busca a freqüência e forma do problema, qualitativamente. $\mathrm{O}$ autor, ao defender uma proposta alternativa para a antropologia médica, cunha o conceito de dispositivo patogênico estrutural, discutindo a necessidade de superação da tendência deformante da antropologia, que explica tudo em termos culturais. Feita esta crítica à sua própria disciplina, Bibeau (op. cit.) assinala sua crítica à epidemiologia, que busca a explicação da enfermidade nos comportamentos de alguns indivíduos, como uso de álcool, tabagismo, ou em características "sociodemográficas", como idade, condições de habitação e identifica os grupos de risco abordando a população como uma agregação de indivíduos, objeto das intervenções dos profissionais. Por outro lado, a antropologia deve buscar a compreensão dos problemas a partir das condições materiais (ambiente ecológico, político-econômico), do contexto social (extensão da rede familiar ou social, ausência ou presença de apoio social) e do contexto cultural (modo de educação, valores, crenças, concepções). Esta disciplina lida com grupos sociais (e não agregado de indivíduos), com sua heterogeneidade social, cultural e econômica, nos quais os atores participam com os profissionais na solução dos problemas (op. cit.).

Embora Bibeau tenha sistematizado essa discussão para sua proposta de uma etnografia limitada de comunidade, como alternativa metodológica para a antropologia médica, ele sinaliza, nesse quadro, aspectos que têm caracterizado a epidemiologia. Ressalvadas suas críticas sobre a sobreculturalização dos problemas pelos antropólogos, conforme referido acima, ele também caracteriza o que têm sido as abordagens qualitativas, especialmente, os estudos antropológicos em saúde.

O texto de Bibeau, ao pontuar o que separa a antropologia da epidemiologia, contribui para identificar alguns aspectos que têm sido apontados por alguns autores como determinantes dos limites da epidemiologia.

Barreto \& Alves (1994), ao discutirem o coletivo e o individual na epidemiologia, e a propósito de sua interface com as ciências sociais, admitem que, embora tenha se estruturado como a disciplina que estuda a distribuição e os determinantes da doença em populações, em um coletivo humano, a epidemiologia enfatiza a determinação de estruturas normativas, padrões de comportamento e valores sociais nos indivíduos. Nesta perspectiva estruturalista, perde de vista a existência de indivíduos concretos que vivenciam situações sociais que lhe são dadas e que interpretam e fornecem significados aos seus comportamentos e aos dos outros. Ou seja, para a epidemiologia, segundo esses autores, a atividade humana resulta das condições materiais coercitivas. Essa perspectiva adotada pela epidemiologia ao lidar com o coletivo, pressupõe, segundo eles, a determinação social sobre o sujeito. Argumentam que, embora haja, de fato, implicações das circunstâncias estruturais, historicamente herdadas, do coletivo, os indivíduos monitoram suas ações em processos interativos, negociando, adaptando e modificando significados e contextos. Para os autores, a epidemiologia, nesta perspectiva, recusa aspectos essenciais da coletividade humana: o universo de significados, motivos, aspirações, atitudes, valores e crenças (op. cit.). A constatação faz os autores enfatizarem a necessidade, já apontada nas ciências sociais, de superação dessas duas perspectivas, em uma síntese que leve em conta a objetividade das estruturas e a subjetividade das práticas individuais.

Mas ao falar em determinação social sobre os indivíduos, não se pode deixar de mencionar a contribuição teórica de autores da epidemiologia social latino-americana que estabeleceram uma ruptura no pensamento médico dominante, cujo postulado fundamental é o caráter natural e a-histórico do processo saúde-doença. Ainda que, na sua perspectiva estruturalista, esses autores estejam vulneráveis a críticas, inclusive algumas dessas formuladas por Barreto \& Alves, já referidas, eles, por sua vez, acumulam o mérito de ter trazido para a epidemiologia uma discussão que elevou seu patamar para além da determinação "natural" da doença.

Ao fazer uma aproximação com esses autores é possível identificar um debate no qual se discutiu os limites das proposições dos grupos da América Latina. 
Nesse debate, Almeida Filho (1993) ressalta a insuficiência de se compreender o processo saúde-doença apenas a partir da categoria processo de trabalho, trazida por Laurell (1983), ou da categoria reprodução social, proposta por Breilh e Granda (1985). Para este autor, isso resultaria em uma redução da complexidade social a uma só dimensão da vida, definindo, assim, um monodeterminismo. Destaca a necessidade de serem consideradas no processo outras dimensões da vida, como o simbólico e a cotidianidade.

Betancourt (1995), tentando ampliar o marco teórico sobre a determinação social e do trabalho no processo saúde-doença, define esse processo como aquele que apesar de se expressar de forma concreta nos indivíduos, corresponde à maneira como se trabalha, como se vive, se alimenta, se educa, descansa, recreia, se organizam os grupos humanos, aos quais pertence o indivíduo. No processo saúde-doença há expressões surgidas na dimensão singular do ser, o qual apesar de corresponder a uma coletividade, apresenta características pessoais, que se diferenciam das dos demais.

Para Lima (1994), Laurell limita-se a afirmar as formas de manifestação coletiva da doença, quando, na verdade, é preciso conhecer a forma e os caminhos específicos pelos quais os processos sociais e determinações gerais se manifestam no indivíduo; conhecer a relação entre o biológico, psicológico inclusive, e o social. Afirma que os estudos realizados por Laurell são situados na generalidade das categorias estruturais, com o indivíduo ocupando um espaço reduzido. Para o autor, o processo de concreção não se esgota nos grupos. A gênese das doenças também envolve dimensões individuais, portanto, necessárias para a explicação e para a prevenção. Isto exige que se compreendam os homens como individualidades sociais, articulando a singularidade biopsíquica e a universalidade genérica em uma unidade complexa (op. cit.).

Ainda em sua crítica à epidemiologia social, Lima (1994) afirma que no caso da sociedade capitalista, abre-se espaço, dentro de determinados limites estruturais, para que os indivíduos tenham comportamentos diferenciados. Estas escolhas não são obviamente livres, mas permitem, ao menos, que os comportamentos concretos se diferenciem, senão em qualidade, certamente em grau. Com isso, diferentes trajetórias de vida, e no caso do processo saúde-doença, diferentes formas de desgaste podem acontecer num leque mais ou menos amplo, que depende também de estratégias individuais no interior das limitações estruturais (op. cit.). A apreensão exclusiva das formas coletivas de adoecer elimina a possibilidade de verificar como o desgaste se estabelece no plano individual, deixando de conhecer processos singulares de saúde-doença, além de eventuais processos práticos de resistência e de transformação já existentes no interior da sociedade, construídos a partir de estratégias individuais ou de pequenos grupos.

Resgatadas algumas contribuições que permearam o debate acerca da epidemiologia social, outras discussões são relevantes para o presente ensaio.

Ao construir sua trajetória de reflexão teórico-epistemológica da epidemiologia, Almeida Filho (1992, 2000, 2000a) escreve e reapresenta uma discussão sobre os paradigmas da epidemiologia, propondo reflexões que conduzam para a superação dos obstáculos contemporâneos da disciplina. Nessa perspectiva, discute os paradigmas existentes, identificando dois paradigmas que não apenas se sucedem, mas coexistem, não sem alguma tensão.

O paradigma I ou paradigma da causalidade é aquele no qual o objeto do conhecimento é a enfermidade, trazida da clínica, daí porque o autor o trata de objeto-semblante: a morbidade de grupos populacionais em correspondência direta à patologia do corpo orgânico individual. Neste paradigma, os nexos do processo de determinação da doença são efeito-específicos e o critério de construção da prova seria a experimentação, o que, segundo o autor, evidencia o paradoxo da sua adoção pela epidemiologia, cuja prática se baseia em desenhos de estudo observacionais, indicados para situações não reprodutíveis sob condições experimentais controladas. $\mathrm{O}$ autor afirma que, apesar do paradoxo, este paradigma está presente nos manuais clássicos da epidemiologia, que adotam, sem muita crítica, os critérios de causalidade. Estes, em última instância, exigem dos desenhos de estudo observacionais o que eles não podem dar, ficando sempre em desvantagem epistemológica diante dos desenhos controlados (ensaios terapêuticos), pretendidos pela clínica ou pela epidemiologia clínica. Almeida Filho fala que a determinação multicausal, apresentada como um avanço da epidemiologia, na verdade, se orienta dentro do mesmo paradigma da causalidade, "alimentada pelo sonho do efeito-específico a ser descoberto pelo avanço científico” (2000).

O paradigma II, paradigma do risco ou próprio da disciplina, tem como objeto de co- 
nhecimento um objeto-resíduo e não o objeto probabilístico, referido nos manuais. Isto porque, para Almeida Filho (1992; 2000), a estatística aqui teria a função de depuração do objeto, ou seja, o que não pode ser explicado por um modelo de distribuição aleatória é o que constitui a determinação epidemiológica; ao ser rejeitada a hipótese nula, aceita-se a hipótese de estudo. O risco, objeto resíduo, é, portanto, a probabilidade de adoecer que se desvia das probabilidades aleatórias (2000). A construção da prova neste paradigma se faz pela inferência preditiva, com o uso da inferência estatística. Os modelos explicativos continuam tendo funções lineares, embora estas se configurem como funções lineares expandidas, admitindo a possibilidade de intercorrelações. No entanto, admite-se as intercorrelações mais no sentido de buscar controlar os efeitos, para fazê-los desaparecer dos modelos de análise (2000). Ou seja, mantém-se o sonho de se chegar à função linear simples, para usar uma palavra referida pelo autor.

Philippe (1998), ao discutir os paradigmas da epidemiologia, cita Laplace, astrônomo do século 18, e seu determinismo, segundo o qual a predição do que vai acontecer pode ser obtida quando as muitas variáveis que controlam o universo forem conhecidas, ou seja, a indeterminação do conhecimento, nesta perspectiva, seria transitória. Além dessa referência, cita a linearidade newtoniana, segundo a qual o resultado de qualquer sistema é conhecido, na medida em que as condições iniciais são especificadas e conhecida uma lei de movimento.

Para Philippe (op. cit.) esta forma de conhecimento está muito presente na epidemiologia: acredita-se que o acréscimo de mais variáveis ao modelo de regressão logística, por exemplo, ajudará a explicar melhor os resultados obtidos, pois se espera que a relação da exposição com o efeito seja linear. Segundo este autor, a prática epidemiológica é, fundamentalmente, newtoniana e laplaceana. Ele discute a não-linearidade dos sistemas, trazendo para a prática da epidemiologia a seguinte questão: a falta de ajuste de um certo modelo de análise em epidemiologia não é o resultado da indeterminação temporária, decorrente da falta de conhecimento sobre os fatores causais, mas pode resultar do determinismo estrutural não-linear. Assim, com base no questionamento sobre a linearidade dos modelos explicativos em epidemiologia, Philippe coloca em dúvida também o pressuposto da independência dos determi- nantes (ou subsistemas explicativos) afirmando que as interações, quando identificadas em um modelo de análise epidemiológica sem ajuste, são utilizadas para forçar o ajuste à linearidade e não para questionar a inadequação dos modelos explicativos lineares. Chama atenção também para os limites do atual paradigma do risco, da inferência preditiva, dos modelos lineares, ainda que expandidos, e para os desafios postos ao campo da epidemiologia para lidar com sistemas dinâmicos, não-lineares.

Para Barreto \& Alves, também, a perspectiva determinística da epidemiologia ao construir modelos empíricos, pretende explicar a totalidade do fenômeno saúde-doença pelo simples acréscimo de novas variáveis, o que, para eles, é insuficiente (1994).

\section{Por uma outra perspectiva: a interdisciplinaridade em construção}

Almeida Filho argumenta, em conformidade com outros teóricos já referidos, que a complexidade de um sistema de explicação de um fenômeno da saúde-doença, cuja determinação é, por certo, irregular, não é dependente do maior número de variáveis explicativas necessárias, mas, sobretudo, dos processos interativos existentes entre essas variáveis. Essa complexidade não admite padrões isolados de determinação, como previa o paradigma I da causalidade, em busca do efeito-específico. Neste sentido, chama a atenção para a necessidade de integração de diferentes perspectivas teóricometodológicas para o estudo da relação saúdedoença. Reconhece os limites da abordagem dos fatores de risco aplicada isoladamente ao estudo desta relação, que requer também modelos capazes de abordar a relação entre sujeitos humanos e seu meio ambiente, cultural e sócio-histórico. Esta relação não trata exclusivamente da ação externa de um elemento ambiental agressivo, conforme indicado na metáfora de fatores-produzindo-riscos, nem da reação internalizada de um hóspede susceptível, senão do sistema (totalizado, interativo, processual) de efeitos patológicos (Almeida Filho, 1992; 1997; 2000; 2000a).

Inhorn (1995) dá uma importante contribuição no sentido de evidenciar as interfaces entre a epidemiologia e antropologia, chamando atenção para o fato de que muitos estereótipos acabam por inibir uma maior integração entre essas disciplinas e a identificação das suas 
convergências. Diz que a visão de parte dos antropólogos de que a epidemiologia adota acriticamente as noções da biomedicina é um desses estereótipos. Para ela, a epidemiologia social assumiu um papel relevante, demarcando um distanciamento crítico com relação à biomedicina. Pontua também a importância do avanço do método epidemiológico em abordar seus vieses, ao passo que a antropologia viveu muito tempo, até recentemente, sob um notável silêncio acerca das limitações potenciais dos empreendimentos etnográficos (op. cit.). A autora cita a opinião dos antropólogos sobre o positivismo e reducionismo da epidemiologia, enquanto se vêem como baluartes do holismo e humanismo, discute as diferenças e aproximações dos métodos adotados pelas duas disciplinas e as diferentes abordagens do risco e o lugar dos sujeitos. Refere que muitas divergências entre as disciplinas são ilusórias e, ao identificar as convergências, releva a necessidade de integração das disciplinas.

Uma questão crucial do limite da abordagem epidemiológica clássica e que vem sustentar a busca pela integração com outras disciplinas pode estar na insuficiência das respostas obtidas através das ações de controle de problemas de saúde, orientadas, exclusivamente, pelos resultados dos estudos quantitativos. Essa questão tem sido apontada por autores de diversos campos, a exemplo de alguns estudiosos da Aids e outras doenças infecto-contagiosas, estudiosos de saúde materno-infantil e tantos outros (Uchoa et al., 2000; Béria et al., 1998; Uchoa \& Vidal, 1994). Os fatores de risco para Aids, objetivamente identificados através dos estudos epidemiológicos, não são suficientes para a compreensão da manutenção da sua ocorrência e distribuição. Ao mesmo tempo e, por conseqüência, as intervenções baseadas apenas nas informações sobre esses fatores não têm permitido um maior êxito das medidas propostas para sua prevenção. Recomendar o uso de preservativos, proscrever o sexo sem proteção, ampliar o acesso à informação sobre as formas de transmissão da doença não têm garantido os resultados esperados entre muitas parcelas da população. Da mesma forma, as campanhas institucionais para a prevenção da esquistossomose foram inefetivas [sic.] em transformar a informação recebida em comportamento preventivo relacionado ao contato com água (Uchoa et al., 2000).

No campo da saúde e trabalho, a busca da integração entre as abordagens qualitativas e quantitativas tem se justificado pelo melhor entendimento que se pode ter do impacto das desordens relacionadas com o trabalho a partir dessa combinação. Mergler (1999) assinala, entre outras vantagens dessa estratégia, a melhor definição das questões de pesquisa, a redução de erros na definição de exposição e de efeitos à saúde, a adequação das intervenções antes do desenvolvimento de doenças bem definidas, além desta prover informações relevantes para melhorar as condições e práticas de trabalho adversas.

O limite da abordagem epidemiológica exclusiva poderia não se constituir em um problema para a produção do conhecimento em si no campo da saúde e trabalho. Entretanto, este é crucial quando a produção de conhecimento objetiva, a partir da identificação dos determinantes da morbi-mortalidade, subsidiar as ações de controle nos ambientes de trabalho. Neste caso, as ações orientadas, exclusivamente, pelos resultados dos estudos quantitativos, horizontais, extensivos, podem ser insuficientes. Se é verdade que há diferentes contextos e relações no mundo do trabalho, um saber-fazer cotidiano, modos operatórios e diferentes competências, é difícil conceber intervenções, ou medidas de controle sobre os riscos no trabalho, as quais, quando implementadas, interferirão no modo operatório dos trabalhadores, sem considerar esses sujeitos na sua atividade cotidiana.

O encontro entre o método epidemiológico, privilegiado para generalização, e outro capaz de aprofundamento pode ser alcançado por meio de diversas iniciativas: as abordagens que buscam a profundidade poderiam validar instrumentos epidemiológicos, particularmente quanto às variáveis independentes. Além disso, dados produzidos por meio dos métodos qualitativos poderiam ser fonte privilegiada na construção de modelos de investigação epidemiológica e, ainda, as técnicas qualitativas poderiam favorecer a formulação de estratégias híbridas que buscassem uma maior aproximação com o real, além da possibilidade do encontro das abordagens qualitativas e quantitativas favorecer a interpretação dos resultados obtidos (Almeida Filho, 2000a).

A construção de modelos interpretativos do processo saúde-doença que integrem perspectivas qualitativas e quantitativas, superada essa dicotomia, pode ser possível a partir de diferentes estratégias. Uma perspectiva tem sido denominada de etnoepidemiologia, que teria $v o$ - 
cação de disciplina aplicada, cujo conhecimento encontraria seu sentido último no processo de transformação das realidades concretas de saúde. Teria como pressuposto que os fenômenos da saúde-doença são processos sociais, devendo ser concebidos como tais, históricos, complexos, fragmentados, conflitivos, dependentes, ambíguos e incertos (Almeida Filho, 1992; 2000).

Almeida Filho (s.d.) sugere três modalidades para a perspectiva etnoepidemiológica: 1) estudo das diversidades étnicas e culturais como fatores de risco, de proteção ou fatores prognósticos para moléstias e outros problemas de saúde; 2) estudos de modelos comunitários de distribuição e ocorrência de doença em populações, ou seja, estudos interessados na ocorrência e prevenção de moléstia em grupos, populações e culturas; 3 ) estudo da prática científica epidemiológica com a aplicação de métodos antropológicos. O autor denomina essas modalidades de etnoepidemiologia tipo I, II e III, respectivamente.

A etnoepidemiologia tipo II parte da compreensão de que as pessoas criam, compartilham, organizam e usam um conhecimento comum, uma semiologia popular e um sistema de signos, que são socialmente e historicamente construídos (Almeida Filho, 2001).

Dentro da mesma perspectiva teórica, uma discussão é trazida por Sevalho \& Castiel (1998) a propósito da necessária aproximação com novas categorias que substituam a enfermidade tomada da clínica. Este conceito é próprio da biomedicina, que despreza as representações, $o$ ponto de vista do paciente e da ordem sociocultural, domínios dos aspectos simbólicos, onde residiria a singularidade mais rica dos indivíduose da cultura. Os autores propõem o termo moléstia, em correspondência a illness, como o conceito capaz de fugir à lógica formal da taxonomia clínico-epidemiológica, na qual o conceito de doença, enfermidade, evoca uma homogeneidade de propriedades e características, pressupondo uma ordem ao fenômeno do adoecimento (op. cit.).

Esse fenômeno da doença ou enfermidade, na perspectiva clínico-epidemiológica, resulta, segundo Sevalho \& Castiel (1998), de modelos de raciocínio causal baseados em uma evolução de eventos, em que os sinais e sintomas são decorrentes de uma entidade construída a partir de processos admitidos como patogênicos, em função de uma etiologia. No entanto, os modelos nem sempre são capazes de definir todos os níveis de eventos, e algumas vezes, di- zem os autores, não é possível ordenar com precisão os fenômenos quanto à etiologia ou mecanismos patogênicos, daí o recurso ao conceito de transtorno, por vezes utilizado, particularmente na área de saúde mental. Concluem que, em função da insuficiência de se lidar com o conceito de enfermidade, o fenômeno adoecimento deve ser pensado em outras bases. Assim, na proposta de uma etnoepidemiologia, que convoca a integração das perspectivas etnológica e epidemiológica, o conceito de moléstia, traduzido por Sevalho \& Castiel (op. cit.) como "sensação difusa de haver algo desagradável, incômodo (perceber-se molesto)", parece ser mais apropriado para apreensão dos efeitos à saúde.

O desafio para o estudo dos processos saúde-doença não se limita aos aspectos teóricos, mas são também localizados nas estratégias metodológicas a serem construídas: Enxergar outras representações de saúde e doença, admitilas na coleta de dados, construir novas taxonomias incorporando a interpretação das narrativas, situá-las no contexto histórico, social e cultural, reconhecer os rituais, perceber a diversidade dos gêneros e grupos sociais no âmbito da singularidade do adoecer humano e considerá-la no coletivo das populações, devem ser algumas questões a serem pensadas (Sevalho \& Castiel, 1998).

A construção da integração de diferentes disciplinas se deparará com questões metodológicas importantes, como a questão da representatividade e seu significado para a epidemiologia e a etnografia, a confiabilidade, as questões éticas, como a impossível neutralidade, o etnocentrismo, sob a utopia da construção de um objeto transdisciplinar. No entanto, este é um dos desafios que está colocado para os pesquisadores que acreditam na possibilidade de uma nova via incorporar o melhor dos campos epidemiológico e antropológico.

\section{Conclusões}

O desenvolvimento de abordagens que integrem a investigação da morbidade e seus determinantes, a partir da metodologia epidemiológica e a identificação, em maior profundidade, dos elementos necessários ao entendimento destas formas de adoecer ou se sentir doente, pode resultar em uma maior contribuição para o conhecimento acerca das possibilidades de promoção da saúde. Isto é o que os problemas de saúde contemporâneos estão a reclamar. Nesta 
perspectiva, a discussão da etnoepidemiologia pode oferecer sustentação teórico-metodológi$\mathrm{ca}$, reservadas as especificidades de cada objeto estudado, para a construção de novas abordagens para o estudo da relação saúde-doença. Permanecem muitos desafios e questões não respondidas sobre as alternativas de integração metodológica e sua superação, certamente, não se dará em um único estudo ou investigação. No entanto, parece possível acumular experiências, que já começam a ocorrer, que viabilizem a construção metodológica e consolidação da integração de disciplinas no campo da saúde.

\section{Referências bibliográficas}

Almeida Filho N 1992. A clínica e a epidemiologia. APCEAbrasco, Salvador.

Almeida Filho N 1992a. Hacia una etnoepidemiología (Esbozo de un nuevo paradigma epidemiológico). Revista de la Escuela de Salud Pública 3(1):33-40.

Almeida Filho N 1993. La práctica teórica de la epidemiología social en América Latina. Salud y Cambio 10(3):25-31.

Almeida Filho N 2000. La ciência tímida. Ensayos de desconstruccion de la epidemiologia. Lugar Editorial, Buenos Aires.

Almeida Filho N 2000a. A ciência da saúde. Hucitec, São Paulo.

Almeida Filho N (s.d.). For an ethnoepidemiology of mental health: theoretical and methodological issues (artigo não publicado).

Almeida Filho N 1997. Transdisciplinaridade e saúde coletiva. Ciência \& Saúde Coletiva 2(1/2):5-20.

Barreto ML \& Alves PC 1994. O coletivo versus o individual na epidemiologia: contradição ou síntese?, pp. 129-135. In Qualidade de vida: compromisso histórico da epidemiologia - Anais do III Congresso Brasileiro de Epidemiologia. Coopmed-Abrasco, Belo Horizonte-Rio de Janeiro.

Béria JU, Damiani MF, Santos IS \& Lombardi C 1998. Physicians' prescribring behaviour for diarrhoea in children: an ethnoepidemiological study in Southern Brazil. Social Science \& Medicine 47(3):341-346.
Betancourt O 1995. Teoria e prática de la salud de los trabajadores. In La salud y el trabajo: reflexiones teoricometodológicas. Monitoreo epidemiológico. Atención básica en salud. Centro de Estudos y Asesoria en Salud (CEAS). Organización Panamericana de la Salud (OPS).

Bibeau G 1992. Hay una enfermidad en las Américas? Outro camino de la antropología médica para nuestro tiempo. VI Congreso de Antropologia en Colombia, Santa Fé de Bogotá.

Breilh J \& Granda E 1985. Os novos rumos da epidemiologia, pp. 241-253. In E Nunes (org.). As ciências sociais em saúde na América Latina. Tendências e perspectivas. OPAS, Brasília.

Dunn F \& Janes C 1986. Introduction: Medical antropology and epidemiology, pp. 3-34. In C Janes, R Stall \& S Gifford (eds.). Antropology and epidemiology: interdisciplinary approaches to the study of health and disease. Reidel Publishing Company, Dordrecht.

Inhorn MC 1995. Medical anthropology and epidemiology: divergencies or convergenies? Social Science \& Medicine 40(3):285-290.

Janes C, Stall R \& Gifford S 1986. Anthropology and epidemiology: interdisciplinary aproaches to the study of health and disease. Reidel Publishing Company, Dordrecht.

Kleinman A 1995. The new wave of ethnographies in medical anthropology, pp. 193-256. In Writing at the 
margin: discourse between anthropology and medicine. University of California Press, Berkeley-Los Angeles.

Kunstadter P et al. 1992. Causes and consequences of increase in child survival rates: ethnoepidemiology among the Hmong of Thailand. Human Biology: an International Record of Research 64(6):821-841.

Laurell AC 1983. A saúde como processo social. In ED Nunes (org.). Medicina social. Global, São Paulo.

Lima F 1994. Ergonomia e LER: como entender o processo de individuação da doença. Fundacentro, Belo Horizonte (Mimeo).

Mergler D 1999. Combining quantitative and qualitative approaches in occupational health for a better understanding of the impact of work-related disorders. Scandinavian Journal of Environmental Health 25 (suppl 4):54-60.

Pereira MG 1995. Epidemiologia, teoria e prática. Guanabara Koogan, Rio de Janeiro.

Philippe P 1998. Teoria do caos e sistemas complexos em epidemiologia. In Teoria epidemiológica hoje: fundamentos, interfaces e tendências. Fiocruz-Abrasco, Rio de Janeiro.

Sallis JF, Owen N \& Fotheringham MJ 2000. Behavioral epidemiology: a systematic framework to classify phases of research on health promotion and disease prevention. Annals of Behavioral Medicine 22(4): 294-298.
Sevalho G \& Castiel LD 1998. Epidemiologia e antropologia médica: a in(ter)disciplinaridade possível. In Antropologia da saúde: traçando identidade e explorando fronteiras. Fiocruz-Relume Dumará, Rio de Janeiro.

Uchoa E et al. 2000. The control of schistosomiasis in Brazil: an ethnoepidemiological study of the effectiveness of a community mobilization program for health education. Social Science \& Medicine 51:1.529-1.541.

Uchoa E \& Vidal JM 1994. Antropologia médica: elementos conceituais e metodológicos para uma abordagem da saúde e da doença. Cadernos de Saúde Pública 10(4):497-504.

Wilbert W \& Haiek G 1991. Phytochemical screening of a Warao pharmacopoeia employed to treat gastrointestinal disorders. Journal of Ethnopharmacology 34 (1):7-11.

Artigo apresentado em 19/2/2003

Aprovado em 11/4/2003

Versão final em apresentada em 8/7/2003 